\title{
Imaging the Awake Visual Cortex with a Genetically Encoded Voltage Indicator
}

\author{
ำ Matteo Carandini, ${ }^{1}$ Daisuke Shimaoka, ${ }^{1,2}$ L. Federico Rossi, ${ }^{1}$ Tatsuo K. Sato, ${ }^{1}{ }^{1}$ Andrea Benucci, ${ }^{1,2 *}$ \\ and $\odot$ Thomas Knöpfel ${ }^{2,3 *}$ \\ ${ }^{1}$ UCL Institute of Ophthalmology, University College London, London EC1V 9EL, United Kingdom, ${ }^{2}$ RIKEN Brain Science Institute, Wako City 351-0198, \\ Japan, and ${ }^{3}$ Division of Brain Sciences, Imperial College London, London SW7 2AZ, United Kingdom
}

Genetically encoded voltage indicators (GEVIs) promise to reveal the membrane potential of genetically targeted neuronal populations through noninvasive, chronic imaging of large portions of cortical space. Here we test a promising GEVI in mouse cortex during wakefulness, a challenging condition due to large hemodynamic activity, and we introduce a straightforward projection method to separate a signal dominated by membrane voltage from a signal dominated by hemodynamic activity. We expressed VSFP-Butterfly 1.2 plasmid in layer $2 / 3$ pyramidal cells of visual cortex through electroporation in utero. We then used wide-field imaging with two cameras to measure both fluorophores of the indicator in response to visual stimuli. By taking weighted sums and differences of the two measurements, we obtained clear separation of hemodynamic and voltage signals. The hemodynamic signal showed strong heartbeat oscillations, superimposed on slow dynamics similar to blood oxygen level-dependent (BOLD) or "intrinsic" signals. The voltage signal had fast dynamics similar to neural responses measured electrically, and showed an orderly retinotopic mapping. We compared this voltage signal with calcium signals imaged in transgenic mice that express a calcium indicator (GCaMP3) throughout cortex. The voltage signal from VSFP had similar signal-to-noise ratios as the calcium signal, it was more immune to vascular artifacts, and it integrated over larger regions of visual space, which was consistent with its reporting mostly subthreshold activity rather than the spiking activity revealed by calcium signals. These results demonstrate that GEVIs provide a powerful tool to study the dynamics of neural populations at mesoscopic spatial scales in the awake cortex.

\section{Introduction}

To understand how the cortex processes sensory information, one must record the activity of large neuronal populations across large portions of cortical space. Ideally, one would monitor all the neurons in a cortical area, and, ideally, in more than one area. However, even a single sensory area in the mouse cortex can extend over several square millimeters. The techniques that record from distinct neurons-microelectrode arrays and twophoton imaging - cannot achieve this spatial coverage. An alter-

Received Feb. 12, 2014; revised 0ct. 26, 2014; accepted 0ct. 31, 2014.

Author contributions: M.C., A.B., and T.K. designed research; M.C., D.S., L.F.R., T.K.S., and A.B. performed research; M.C., D.S., L.F.R., and A.B. analyzed data; M.C., A.B., and T.K. wrote the paper.

This work was supported by the Wellcome Trust and the European Research Council. D.S. was supported by a fellowship from the Japanese Society for Promotion of Science, and L.F.R. was supported by a Wellcome Trust studentship. M.C. holds the GlaxoSmithKline/Fight for Sight Chair in Visual Neuroscience. We thank Charu Reddy for outstanding technical support, I-Chun Lin and Michael Okun for recording electrophysiological signals, and the members of the former laboratory for Neuronal Circuit Dynamics at the RIKEN Brain Science Institute for help with preliminary experiments.

The authors declare no competing financial interests.

*A.B. and T.K. contributed equally to this work.

This article is freely available online through the J Neurosci Author Open Choice option.

Correspondence should be addressed to Daisuke Shimaoka or Matteo Carandini, UCL Institute of Ophthalmology, University College London, 11-43 Bath Street, London EC1V 9EL, UK. E-mail: d.shimaoka@ucl.ac.uk or m.carandini@ucl.ac.uk.

DOI:10.1523/JNEUROSCI.0594-14.2015

Copyright $\odot 2015$ Carandini et al.

This is an Open Access article distributed under the terms of the Creative Commons Attribution License (http://creativecommons.org/licenses/by/3.0), which permits unrestricted use, distribution and reproduction in any medium provided that the original work is properly attributed. native is wide-field optical imaging, which covers millimeters of cortical space. This mesoscopic approach has been successfully applied with voltage sensitive dyes (VSD), particularly in visual and somatosensory areas, and has contributed much to the understanding of cortical circuit dynamics (Grinvald and Hildesheim, 2004).

Recently developed genetically encoded voltage indicators (GEVI) promise to improve upon classic VSDs in multiple ways (Knöpfel, 2012), as follows: (1) in species with a thin cranium, such as mice, they allow for noninvasive imaging; (2) they allow genetic targeting to specific cell populations, providing a handle into cellular diversity; (3) they provide reliable recordings, from those cell populations over prolonged periods and multiple sessions; and (4) they enable transgenic expression strategies for highly reproducible delivery of the indicator.

However, like most indicators, GEVIs have optical spectra that overlap with the absorption spectrum of hemoglobin (Hillman, 2007). The intensity of their fluorescence measured through blood-perfused tissue, therefore, is corrupted by vascular signals. These signals reflect changes in local blood volume and oxygenation that are associated with heart beats and with hemodynamic responses. The latter are particularly large and fast during wakefulness (Pisauro et al., 2013, and references therein), thus posing a considerable challenge.

Here we test a highly sensitive GEVI, VSFP-Butterfly 1.2 (Akemann et al., 2010, 2012), in the visual cortex of awake mice, and we show that it yields strong voltage signals, which can be 

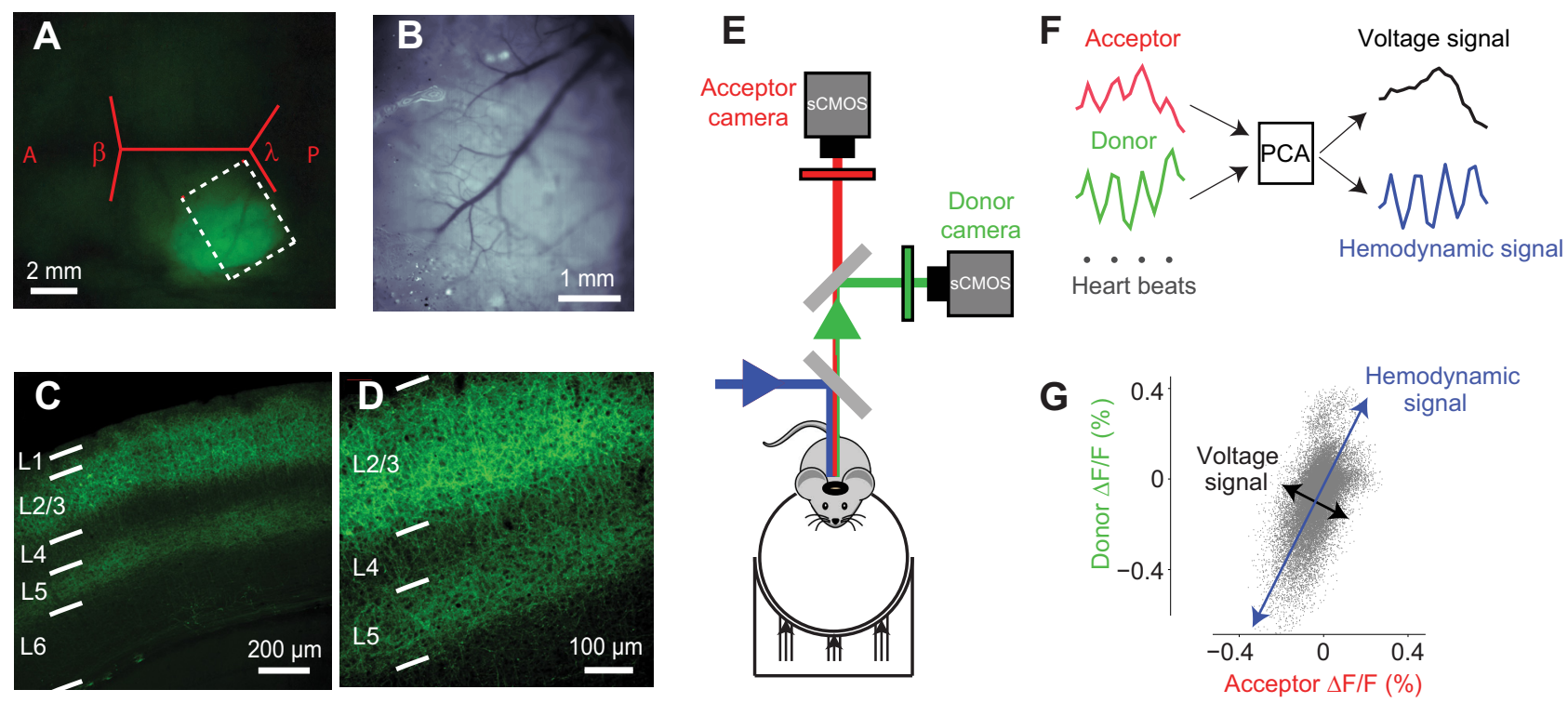

Figure 1. Imaging VSFP fluorescence from layer $2 / 3$ pyramidal cells in mouse visual cortex. $A$, Wide-field imaging of mCitrine fluorescence on the left hemisphere through the thinned bone. White rectangle, Region of interest for subsequent chamber implantation and fluorescence imaging. A-P, Anterior-posterior. $\boldsymbol{B}$, Imaging of the vascularization in the same Rol under green illumination, through the chamber. $\boldsymbol{C}, \boldsymbol{D}$, Confocal images showing VSFP-Butterfly 1.2 expression pattern at low $(\boldsymbol{C})$ and high $(\boldsymbol{D})$ magnification. $\boldsymbol{E}$, The imaging setup, showing the two $\mathrm{SCMOS}$ cameras, the light path, and the spherical treadmill. $\boldsymbol{F}$, Principal component analysis (PCA) of the mCitrine and mKate2 intensities to extract voltage and hemodynamic signals. $\mathbf{G}$, The method works by projecting the donor and acceptor intensities on to the two principal axes of variability. Dots represent the relative fluorescence $(\Delta \mathrm{F} / \mathrm{F})$ of different pixels at different moments in time. Projection onto the first axis yields hemodynamic signals (blue), and projection onto the second axis yields voltage signals (black).

readily and fruitfully separated from hemodynamic signals. This GEVI exhibits voltage-dependent Förster resonance energy transfer (FRET; Romoser et al., 1997; Jares-Erijman and Jovin, 2003) between a pair of green and red fluorophores: mCitrine (donor) and mKate2 (acceptor). By imaging both fluorophores and combining their fluorescence in a simple linear fashion, we were able to separate a voltage signal and a hemodynamic signal.

Both signals were large compared with the measurement noise. The voltage signal had fast temporal dynamics, similar to those observed with intracellular recordings and with classic VSD imaging. The hemodynamic signal, instead, had both fast dynamics dominated by heart beats, and slow temporal dynamics similar to blood oxygenation level-dependent (BOLD) signals measured with functional MRI (Desai et al., 2011) and with optical imaging (Pisauro et al., 2013).

The voltage signal had a signal-to-noise ratio similar to that of signals measured with wide-field imaging of a calcium indicator (GCaMP3; Zariwala et al., 2012). The voltage signal, however, was more immune from vascular artifacts, and it presents the advantage of reporting not only spiking activity (as for calcium signals), but also subthreshold inputs.

\section{Materials and Methods}

All experimental procedures were conducted according to the UK Animals Scientific Procedures Act (1986). Experiments were performed at University College London under personal and project licenses released by the Home Office following appropriate ethics review.

In utero electroporation. To achieve strong expression, we delivered the indicator gene by in utero electroporation (Fukuchi-Shimogori and Grove, 2001; Saito and Nakatsuji, 2001) under the ubiquitous CAG promoter. To target cells destined for layer $2 / 3$, we performed the procedure at embryonic day 15.5. Pregnant mice (C57BL/6J) were anesthetized with $2 \%$ isoflurane in oxygen. Up to $1 \mu \mathrm{l}$ of DNA solution with Fast Green $(0.05 \% \mathrm{w} / \mathrm{v}$; Sigma $)$ was pressure injected into one lateral ventricle of embryos. The solution contained pCAG-VSFP-Butterfly 1.2 plasmid ( 5 $\mu \mathrm{g} / \mu \mathrm{l}$, Akemann et al., 2012). Electroporation was achieved with five square pulses ( $50 \mathrm{~V}, 50 \mathrm{~ms}, 1 \mathrm{~Hz}$; CUY21, NepaGene). The pulses were targeted to the occipital region of the injected hemisphere (Mizuno et al., 2007; Sato et al., 2014). We used mCitrine fluorescence (wavelength, $535 \pm 30 \mathrm{~nm}$ ) to screen for positive animals under a fluorescence dissecting scope (MVX10, Olympus) on the day of birth. This fluorescence confirmed clear expression in a cortical region including area V1 (Fig. $1 A, B)$.

Surgery. Electroporated mice (1-3 months old) of either sex were implanted with a head plate and a circular window for imaging through thinned bone. An analgesic (Rimadyl) was administered ( $0.05 \mathrm{ml}$, s.c.) on the day of the surgery, and on subsequent days as needed. Surgery was performed under sterile conditions. The animal was anesthetized with isoflurane $(2 \%)$. Body temperature was kept at $37-38^{\circ} \mathrm{C}$, and the eyes were protected with ophthalmic gel (Viscotears Liquid Gel, Alcon). The head was shaved and disinfected, the cranium was exposed, the bone was thinned with a scalpel over the visual cortex, and a head plate was secured with dental cement. The plate has a round opening ( $5 \mathrm{~mm}$ in diameter) for optical imaging of the visual cortex. After the cement solidified, this opening was filled with cyanoacrylate and covered with a round coverslip. The skin was sutured around the implant where necessary.

Visual stimuli. Visual stimuli were presented on two LCD monitors (60 $\mathrm{Hz}$ refresh rate; MultiSync LCD2190UX, NEC) arranged to cover $90^{\circ}$ of visual field contralateral from the imaged hemisphere and positioned $\sim 30 \mathrm{~cm}$ from the animal. For mapping retinotopy, stimuli were contrastreversing gratings presented inside a rectangular window. Stimulus duration was $5 \mathrm{~s}$, flickering frequency was $2 \mathrm{~Hz}$, and spatial frequency was 0.03 cycles $/{ }^{\circ}$. To measure preferred azimuth, the rectangular window was $60^{\circ}$ high and $20^{\circ}$ wide. To measure preferred elevation, it was $20^{\circ}$ high and $60^{\circ}$ wide. When studying the propagation of activity in visual cortex, the stimulus was a bar flashed for $200 \mathrm{~ms}$. We occasionally monitored eye movements with a camera and found them to be rare (one every few seconds), small (on the order of $10^{\circ}$ ), and restricted to the horizontal dimension (Ayaz et al., 2013).

Electrophysiology. In five additional mice, we performed electrophysiological recordings of the responses to flashed bars. We inserted silicon probes (NeuroNexus): either two shanks with 16 sites each (covering 750 $\mu \mathrm{m}$; i.e., almost all layers) or four shanks with 8 sites each (aimed at layer 5). Signals were amplified and sampled at $30 \mathrm{kHz}$ (BlackRock) and then filtered between 0.1 and $100 \mathrm{~Hz}$ to obtain local field potentials (LFPs). 
Multiunit activity (MUA) was obtained by nonlinear high-pass filtering of the original traces, followed by thresholding (Hazan et al., 2006) and spike sorting to distinguish spikes from noise and avoid double counting of spikes appearing simultaneously in multiple electrode sites.

VSFP imaging. To monitor the FRET between the mCitrine-mKate2 fluorophore pair, we used an epi-illumination system (Akemann et al., 2010; Fig. 1E). We excited mCitrine with a blue LED light (LEX2-B, Brain Vision) or with a tungsten-halogen lamp passed through a bandpass filter (FF01-482/35-25, Semrock). The excitation light was diverted onto the visual cortex via a dichroic mirror (FF506-Di03, Semrock). The emitted fluorescence from the donor (mCitrine; Fig. $1 E$, green) was reflected by a second dichroic mirror (FF593-Di03, Semrock), passed through an emission filter (FF01-543/50-25, Semrock), and collected by a scientific complementary metal oxide semiconductor (sCMOS) camera (pco.edge, PCO AG). The emitted fluorescence from the acceptor (mKate2; Fig. $1 E$, red) was transmitted by the second dichroic mirror, passed through an emission filter (BLP01-594R-25, Semrock), and collected by a second sCMOS camera. Cameras were controlled by a TTL external trigger synchronized with the visual stimulation. To obtain a baseline fluorescence level, the camera acquired frames for $1 \mathrm{~s}$ before each stimulus trial. The image acquisition rate was $60 \mathrm{~Hz}$, with a nominal spatial resolution of $200 \mathrm{pixel} / \mathrm{mm}$. Frames from the two cameras were aligned off-line using the Image Processing Toolbox in Matlab.

Equalized ratio. To obtain a ratiometric estimate of membrane potential in each pixel, we used the standard equalized ratio approach (Akemann et al., 2012; Knöpfel, 2012). In this approach, the fluorescence intensities of the acceptor $A(x, y, t)$ and donor $D(x, y, t)$ are scaled by a gain factor that corrects for wavelength-dependent light absorption by hemoglobin, as follows:

$$
\begin{aligned}
& A_{e}=\gamma(A-\bar{A})+\bar{A}, \\
& D_{\mathrm{e}}=\delta(D-\bar{D})+\bar{D},
\end{aligned}
$$

where $\bar{A}(x, y)$ and $\bar{D}(x, y)$ are baseline intensities (typically, averages over time in intervals that precede a stimulus), and $\gamma$ and $\delta$ are scalar correction factors for the acceptor and donor calculated as follows:

$$
\gamma=\left(1+\frac{\bar{A} \sigma_{D}}{\bar{D} \sigma_{A}}\right) / 2, \delta=\left(1+\frac{\bar{D} \sigma_{A}}{\bar{A} \sigma_{D}}\right) / 2,
$$

where $\sigma_{A}(x, y)$ and $\sigma_{D}(x, y)$ represent the SDs of $A$ and $D$ measured in the frequency band of the heart rate (Akemann et al., 2012), where these fluorescence measurements are dominated by correlated fluctuations of hemoglobin absorption. The gain-corrected ratiometric signal is calculated as follows:

$$
\frac{\Delta R}{\bar{R}}=\frac{A_{e}}{D_{e}} \frac{\bar{D}_{e}}{\bar{A}_{e}}-1
$$

This signal reflects membrane voltage, discounting the changes in hemoglobin concentration and oxygenation that lie in the optical path.

Projection method. To estimate hemodynamic activity and separate it from voltage activity, we developed an alternative method, which does not involve equalizing the contributions of the two fluorophores. We computed the relative intensity of the acceptor and donor channels $a(x, y, t)=\frac{\Delta A}{\bar{A}}$ and $d(x, y, t)=\frac{\Delta D}{\bar{D}}$. We then combined the two into a $N$ by 2 matrix $(a, d)$, where $N$ represents the concatenated pixels and time points, and we performed principal component analysis to obtain two two-dimensional vectors, the principal components (Fig. $1 F, G$ ). The first vector, $C_{1}$, reflects congruent temporal fluctuations of the two intensities. The second vector, $C_{2}$, isolates intensity changes with opposite polarity. We thus projected the recorded intensities onto those two axes, and obtained estimates of hemodynamic and voltage activity at each pixel and time, $C_{1}(x, y, t)$ and $C_{2}(x, y, t)$.
Because the intensities $a$ and $d$ are small, the voltage signal obtained through this method is similar to the ratiometric estimate. Indeed, a Taylor expansion for Eq. 3 yields the following:

$$
\begin{gathered}
\frac{\Delta R}{\bar{R}}=\frac{\gamma}{\bar{A}}(A-\bar{A})-\frac{\delta}{\bar{D}}(D-\bar{D})+\ldots=\frac{\bar{A} \sigma_{D}+\bar{D} \sigma_{A}}{2 \bar{A} \bar{D}}\left(\frac{A}{\sigma_{A}}-\frac{D}{\sigma_{D}}\right) \\
-\gamma+\delta+\ldots,
\end{gathered}
$$

and the second principal component can be written as follows:

$$
C_{2}=\frac{\sqrt{2}}{2}\left(\frac{A}{\sigma_{A}}-\frac{D}{\sigma_{D}}\right)+\text { const } .
$$

Hence, $\frac{\Delta R}{\bar{R}}$ and $C_{2}$ both grow approximately linearly with $\left(\frac{A}{\sigma_{A}}-\frac{D}{\sigma_{D}}\right)$. The above derivation assumes that the principal components were obtained on traces filtered to isolate the heartbeat frequency, as is done for the ratiometric quantities. In practice, we found that performing or omitting this filtering step led to similar principal component axes, consistent with the fact that heart beats dominate the raw acceptor and donor signals.

Imaging of calcium signals. We generated mice expressing GCaMP3 by crossing the following two transgenic lines: Emx1-IRES-Cre, expressing Cre recombinase under the Emx1 promoter (catalog \#005628, The Jackson Laboratory); and a reporter Ai38-GCaMP3 line (catalog \#014538, The Jackson Laboratory), carrying a floxed copy of the GCaMP3 gene under the strong CAG promoter in the Rosa26 locus. Offspring expressed GCaMP3 in excitatory neurons of the neocortex and hippocampus, and in the glial cells of the pallium (Gorski et al., 2002). GCaMP3 fluorescent signals were imaged through the filters and camera used for mCitrine when imaging VSFP signals (see above).

Confocal imaging. To prepare the brain for tissue section, the animal was anesthetized (pentobarbital sodium, $200 \mathrm{mg} / \mathrm{kg}$, i.p.), and the heart was perfused with PBS $(0.5 \mathrm{ml} / \mathrm{min})$ followed by fixative (paraformaldehyde, $4 \%$ ) in PBS solution. The brain was removed and immersed in a PBS containing $30 \%$ sucrose at $4^{\circ} \mathrm{C}$. Frozen coronal sections $(60 \mu \mathrm{m}$ thick) were obtained with a sliding microtome (Microm HM400R) and were later viewed through a confocal microscope.

\section{Results}

We expressed VSFP-Butterfly 1.2 plasmid in mouse primary visual cortex (V1) and selectively targeted it to layer 2/3 pyramidal cells through in utero electroporation (Fig. $1 A-D$ ). Confocal imaging confirmed that VSFP expression was predominant in the soma, dendrites, and axons of layer $2 / 3$ pyramidal cells (Fig. $1 C, D)$. This highly preferential expression pattern was present in both young and adult mice. Once the animals were adult, we implanted a head post with a circular window that allowed fluorescence imaging through the thinned bone. We were then able to repeatedly image the mice, while they were awake, head fixed, and free to run on a spherical treadmill (Dombeck et al., 2007) placed in front of visual stimuli (Fig. $1 E$ ).

\section{Ratiometric estimate of voltage signals}

A common difficulty when imaging fluorescence from sources within the brain is that the path between the cameras and the fluorophores goes through tissue that contains blood (Knöpfel, 2012). To a first approximation, this tissue acts as a time-varying optical filter, whose absorption coefficient fluctuates with heart beats and hemodynamic activity. Indeed, changes in blood volume and oxygenation affect the optical properties of the tissue at wavelengths where hemoglobin has a high absorption coefficient (400-600 nm).

VSFP-Butterfly 1.2 plasmid can substantially reduce this problem because it is based on FRET (Jares-Erijman and Jovin, 2003): a change in membrane potential has opposite effects on 


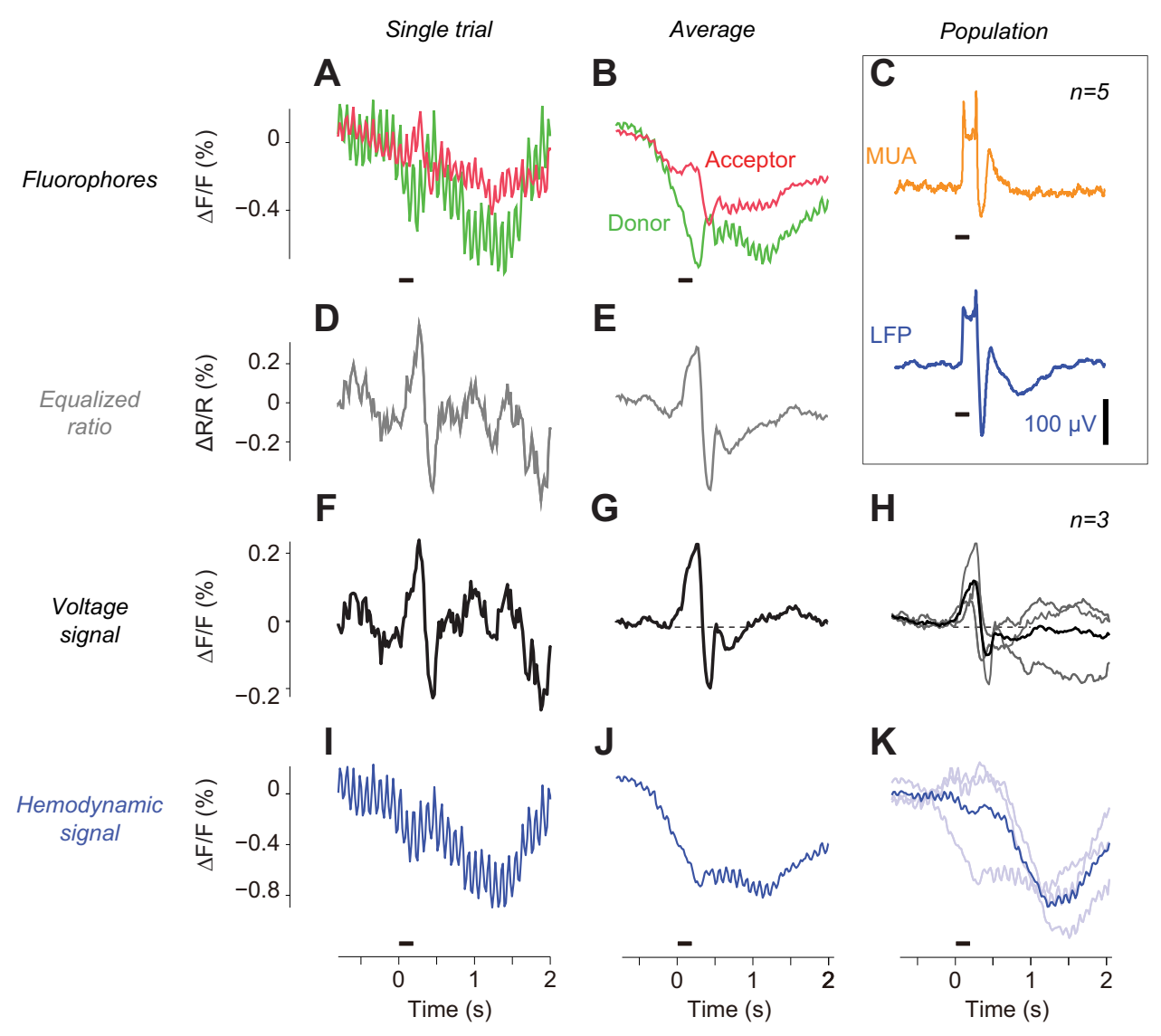

Figure 2. Separation of hemodynamic and voltage signals in response to brief visual stimuli. $A$, Fluorescence intensities measured by the two cameras following a briefly presented grating (one cycle of a square wave grating, 0.03 cycles $/^{\circ} ; 70 \times 20^{\circ} ; 65^{\circ}$ eccentricity), flashed at time 0 for $200 \mathrm{~ms}$ (black bar). $\boldsymbol{B}$, Same as $\boldsymbol{A}$, averaged over 20 stimulus trials. C, LFP and MUA averaged over 100 trials of the same stimulus, in area $V 1$ of five mice. $\boldsymbol{D}, \boldsymbol{E}$, The voltage signal estimated through the ratiometric approach from the traces in $\boldsymbol{A}$ and $\boldsymbol{B}$. $\boldsymbol{F}, \boldsymbol{G}$, Voltage signals obtained by the projection method from the traces in $\boldsymbol{A}$ and $\boldsymbol{B}$. $\boldsymbol{H}$, Voltage signals obtained in three mice (gray) and averaged across animals (black). Each trace is the averaged over $20-30$ trials. $\boldsymbol{I}, \boldsymbol{J}$, Hemodynamic signal estimated by the projection method from the traces in $\boldsymbol{A}$ and $\boldsymbol{B} . \boldsymbol{K}$, Hemodynamic signals obtained in three mice (light blue) and averaged across animals (blue).

the fluorescence of the donor (mCitrine) and the acceptor (mKate2). It can be measured by taking the ratio of their fluorescence. This ratio discounts the vascular factors, as those affect the fluorescence of the two fluorophores in an approximately proportional manner. This method, however, has been validated only under anesthesia (Akemann et al., 2012), where hemodynamic activity is typically much smaller and slower than in wakefulness (Pisauro et al., 2013). We thus first asked whether it works in wakefulness.

As expected, when we measured the fluorescence of the two fluorophores in the awake mouse, we found it to be strongly influenced by hemodynamic factors (Fig. 2A-C). Following a brief visual stimulus, the two fluorescence traces (averaged across pixels located in area V1) shared a slow decrease superimposed on large pulsations associated with the heart beats (Fig. 2A). These effects are signatures of changes in blood volume and oxygenation, which are associated with circulation and neurovascular coupling. Their sources are mostly optical, rather than mechanical, because they persisted after motion compensation (data not shown). Repeating the measurement multiple times and aligning by stimulus onset averaged out the large pulsations (because heart beat and stimulus were not phase locked) and revealed clear stimulus-triggered hemodynamic responses that continued for many seconds after the stimulus (Fig. 2B). These slow responses do not directly reflect neuronal activity: in awake mice, the neural responses to these stimuli constitute an approx- imately triphasic trace that lasts only a few hundred milliseconds (Fig. 2C).

Reassuringly, the standard ratiometric approach recovered a voltage signal that closely resembled the neural responses measured with microelectrodes (Fig. 2D,E). The equalized ratio method estimates the voltage signal by scaling the two traces and taking their ratio (Akemann et al., 2012). When we applied this method to single trials, we found fast biphasic responses, which were only minimally contaminated by the heart beat optical artifact (Fig. 2D). Averaging across trials further reduced this artifact, leaving a trace that resembled the neural responses recorded with electrodes (Fig. 2C). These results indicate that the ratiometric method succeeds not only in the anesthetized cortex (Akemann et al., 2012), but also in the awake cortex.

\section{Projection estimates of voltage and hemodynamic signals}

To better interpret VSFP measurements, we devised a method to extract the hemodynamic signals so that they can be investigated in addition to the voltage signals (Fig. $1 F, G$ ). We reasoned that hemodynamic signals would result in positively correlated measurements in the two emission channels (the two cameras), whereas anticorrelated measurements reflect changes in membrane potential. We thus developed a simple projection method to obtain a hemodynamic signal and a voltage signal (Fig. $1 F$ ). The method is an alternative to the ratiometric method. It works by identifying the axes of maximal correlation and anticorrela- 

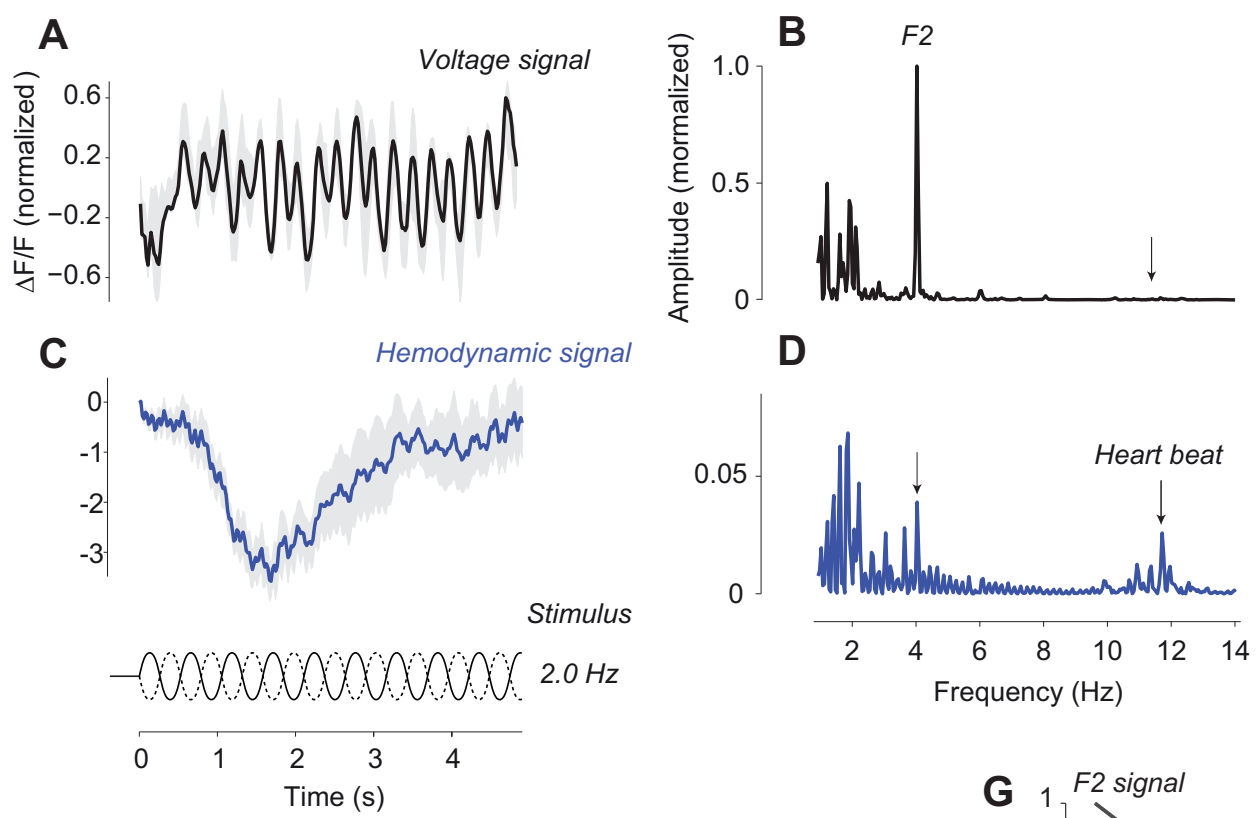

E
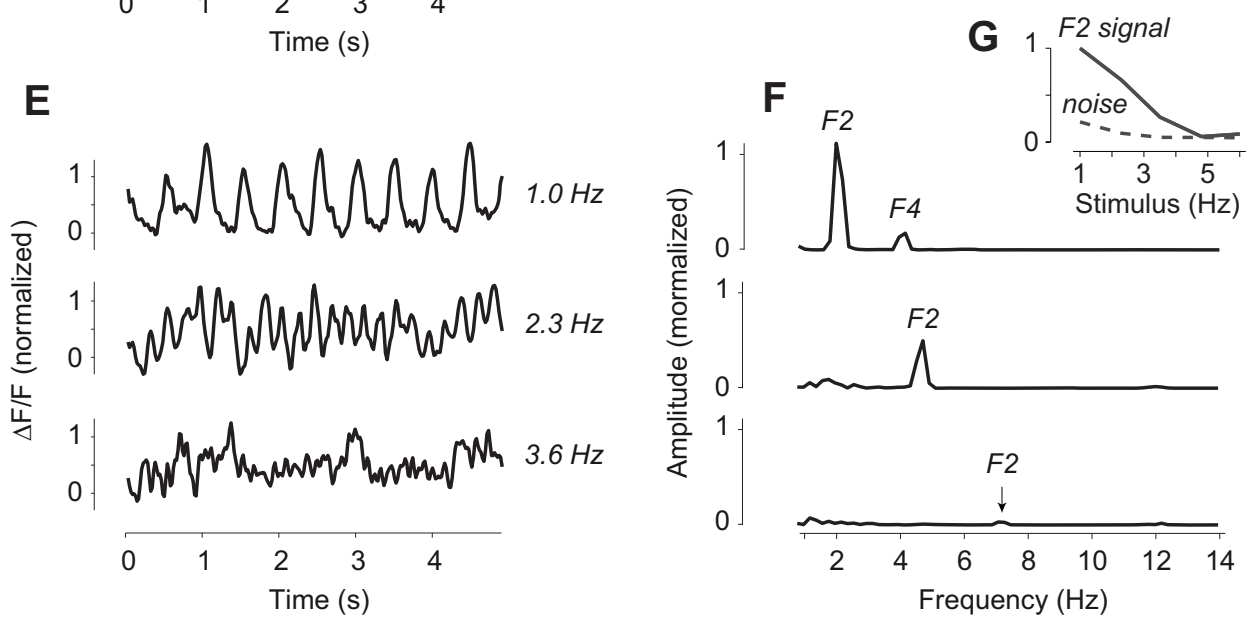

Figure 3. VSFP responses to periodic stimuli. $\boldsymbol{A}$, Voltage signal, averaged over six stimulus repetitions. The stimulus was a vertical rectangular grating, positioned at $65^{\circ}$ eccentricity in the contralateral visual field: height $80^{\circ}$; width, $30^{\circ}$; half white, half black; contrast reversing at $2 \mathrm{~Hz}$. Shaded gray region indicates the $\mathrm{SD} ; n=6$ stimulus repetitions. $\boldsymbol{B}$, Amplitude spectrum of the trace in $\boldsymbol{A}$. Traces are truncated at $0.7 \mathrm{~Hz}$ to improve the visibility of higher-frequency peaks. F2 is twice the stimulus frequency (second harmonic). $\boldsymbol{C}, \boldsymbol{D}$, Same as $\boldsymbol{A}$ and $\boldsymbol{B}$, for the hemodynamic signal, averaged over the same six stimulus repetitions. $\boldsymbol{E}$, Same as $\boldsymbol{A}$, for the following three stimulus frequencies: $1.0,2.3$, and $3.6 \mathrm{~Hz}$. $F$, Amplitude spectra of the traces shown in $\boldsymbol{E}$. $\boldsymbol{G}$, Amplitude of second harmonic responses ( $F 2$, normalized to peak), as a function of stimulus frequency, averaged over six stimulus trials in three animals, in response to flickering gratings ("signal," solid) and blank stimuli ("noise," dashed).

tion (which we perform through principal component analysis) and then projecting the data on those two axes (Fig. 1G). Projection is a simple linear operation, a weighted sum with weights determined by the axis of interest.

To test this projection method, we first confirmed that it gives appropriate estimates of the voltage signal (Fig. $2 \mathrm{~F}-\mathrm{H}$ ). In individual trials, the voltage signal estimated by the projection method was fast and biphasic (Fig. $2 F$ ), with negligible contamination by heart beat artifacts. This contamination was further reduced when averaging across trials, revealing a biphasic trace that fully returned to baseline within a few hundred milliseconds (Fig. 2G). These estimates of the voltage signal are very similar to those made with the ratiometric method (Fig. $2 D, E$ ). Indeed, the Pearson correlation in the traces measured with the two methods was high ( $r=0.90, p<10^{-55}, N=154$ time points).

We then examined the hemodynamic signal, and found that it had all the characteristics that would be expected from changes in blood volume and oxygenation (Fig. $2 I, J$ ). In response to single trials, it contained both a strong component oscillating with the heart rate, and a slow hemodynamic response to the stimulus (Fig. 2I). As expected, the fast oscillation was greatly reduced when averaging across trials, revealing a slow trace that peaked $\sim 1 \mathrm{~s}$ after the stimulus (Fig. $2 J$ ), which is broadly consistent with measurements of blood volume in the cortex of awake mice (Pisauro et al., 2013). In addition to the response to the stimulus, this trace exhibited remains of the responses to previous stimuli, as well as slow drifts typical of hemodynamic signals. Similar results were seen in two other mice, with average voltage signals fast and biphasic (Fig. $2 \mathrm{H}$ ), and hemodynamic signals slow and negative (Fig. $2 \mathrm{~K}$ ). Because the projection method treats each time point independently, this difference suggests good separation of the two signals.

\section{Separation of voltage and hemodynamic signals}

We have seen that the projection method provides a simple and intuitive way to estimate not only the voltage signal but also the hemodynamic signal. The previous analysis indicates good separation of these signals, but does not rule out the possibility 

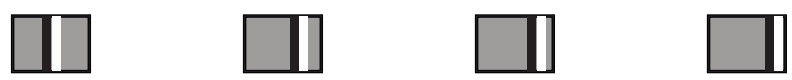

Hemodynamic signal
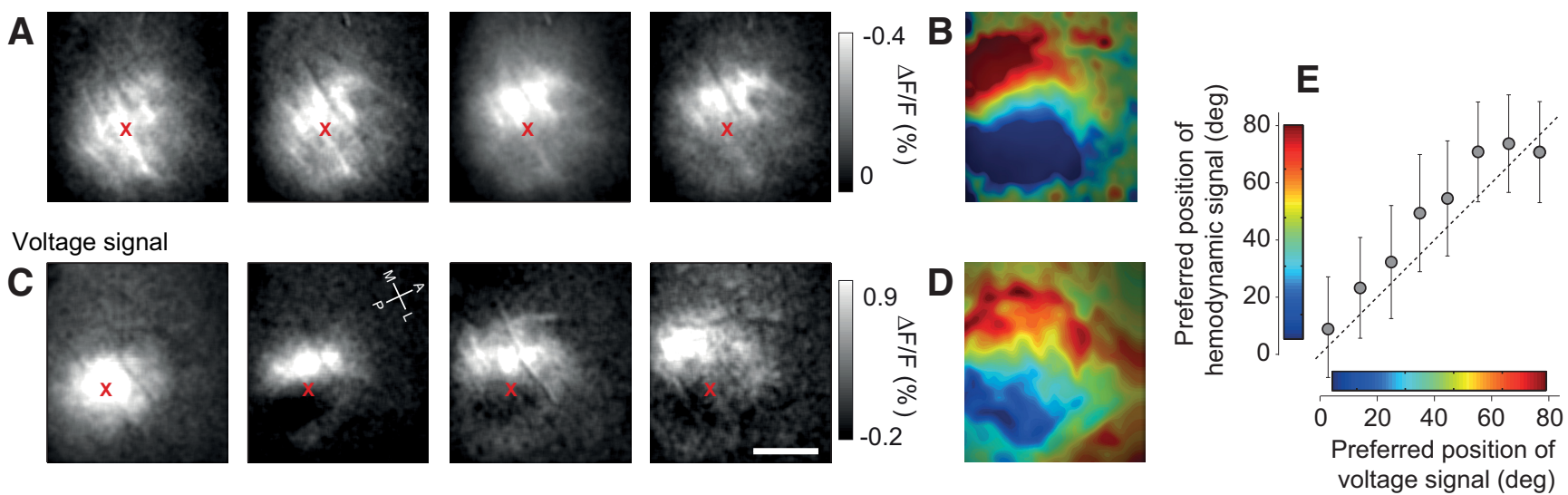

Figure 4. Maps of retinotopy. Stimuli were vertical bars presented in four horizontal positions (top). $\boldsymbol{A}$, Cortical localization of the hemodynamic signal with azimuths of changing stimuli. White indicates the amplitude of negative responses, and the color map is the same for all four panels. Red cross marks a reference point to help compare activations across panels. The scale bar indicates negative numbers because these images correspond to darkening of the cortex. $B$, Map of retinotopy for the hemodynamic signal shown in $A$. Preferred stimulus position is indicated with a color code (scale bars in $\boldsymbol{E}$ ). C, Same as $\boldsymbol{A}$, but for the voltage signal (amplitude of the second harmonic response to contrast reversal). D, Maps of retinotopy for the voltage signal. Color intensity indicates the strength of the tuning. $E$, Correlation of position tuning between hemodynamic signal and the voltage signal (error bars indicate SE; $n=4$ animals).

of cross-contamination. In fact, some level of contamination is present, because the voltage signal in single trials shows a small but visible oscillation at the frequency of the heart rate (Fig. $2 F)$.

To estimate these contaminations, we elicited periodic neural responses, so that each contribution could be tagged with a frequency and, thus, measured (Fig. $3 A-D$ ). We elicited periodic neural responses with gratings whose contrast reversed sinusoidally over time. These stimuli elicit neural responses that oscillate at twice the frequency of reversal (second harmonic), largely because complex cell responses are independent of spatial phase (Movshon et al., 1978). Simple cells, instead, respond to these stimuli at the frequency of reversal (first harmonic), but with two possible phases depending on the spatial profile of the receptive field. If these two phases are equally prevalent, these responses will cancel each other. The resulting oscillating responses at the second harmonic frequency have been measured with multiple techniques, including VSD imaging (Benucci et al., 2007).

Consistent with expectations, the voltage signal contained a strong oscillation at the second harmonic frequency, with negligible amplitude at the frequency of the heart beat (Fig. $3 A, B$ ). The hemodynamic signal, instead, was dominated by the slower response (Fig. 3C) that is seen when imaging blood volume (Pisauro et al., 2013). Superimposed on this slow response was a small contamination from the oscillating neural response. This contamination indicates that the voltage signal underestimates the oscillating neural response, as some of this response makes its way into the putative hemodynamic signal. The amplitude of this contamination, however, was $>60$ times smaller than that of the slow hemodynamic response, confirming that the putative hemodynamic signal was indeed dominated by hemodynamic activity (Fig. 3C).

As expected from neural responses in visual cortex (Van den Bergh et al., 2010; Andermann et al., 2011; Marshel et al., 2011), the amplitude of the oscillating voltage response decreased with stimulus frequency, with a noticeable reduction when stimulus frequency went above $3 \mathrm{~Hz}$, corresponding to neural activity oscillating at $6 \mathrm{~Hz}$ (Fig. $3 E, F)$. Similar results were seen in the other two mice (Fig. 3G). In subsequent experiments involving contrast-reversing stimuli, therefore, we generally chose frequencies of 1 or $2 \mathrm{~Hz}$. We emphasize, however, that these choices reflect the sluggish nature of visual responses, not a limitation of VSFP, which has much higher temporal resolution (Akemann et al., 2012).

In summary, contrast-reversing stimuli elicited strong oscillating responses in the voltage signal but only very weak ones in the hemodynamic signal. Conversely, as we have seen from the analysis of single trials, the heart beat effects are preponderant in the hemodynamic signal and are barely visible in the voltage signals (Fig. $2 F$ ). These results confirm that there is limited contamination between the two sources, as follows: the voltage signal is primarily neural, and the hemodynamic signal is primarily vascular.

Though we did not explore longitudinal imaging in any formal way, we generally found that mice could be imaged multiple times after implantation. For instance, a mouse that was imaged four times after implantation, using the same contrast-reversing gratings and over the same region of interest, gave consistently robust responses for $40 \mathrm{~d}$ after implantation. We compared the amplitude of second harmonic responses (averaged over six stimulus trials) in the presence of the grating (signal) and in its absence (noise). The strength of the signal was highest in the first session (signal-to-noise ratio, 4.7 at day 4), but the quality was high enough that responses could be obtained as far as $40 \mathrm{~d}$ after implantation (signal-to-noise ratio, 2.8). These results were fairly typical and in line with those obtained with genetically encoded calcium indicators (GECIs; Zariwala et al., 2012). The main limitation seemed to be the clarity of the optical window. Indeed, previous studies (Akemann et al., 2010, 2012) based on intracellular electrophysiology indicate that cells expressing VSFP are healthy in adult mice that were transfected with similar methods.

\section{Retinotopy of voltage and hemodynamic signals}

Having established that voltage signals and hemodynamic signals are functionally distinct, and that their temporal profiles are very different, we investigated their spatial profile (Fig. 4). We used periodic stimuli, so that we would elicit strong oscillating neural 
A

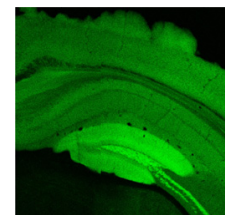

B

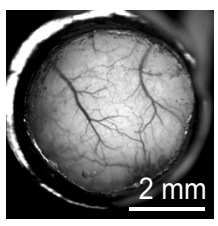

C

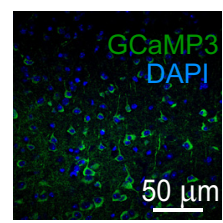

Stimulus vivingniving

GCaMP3

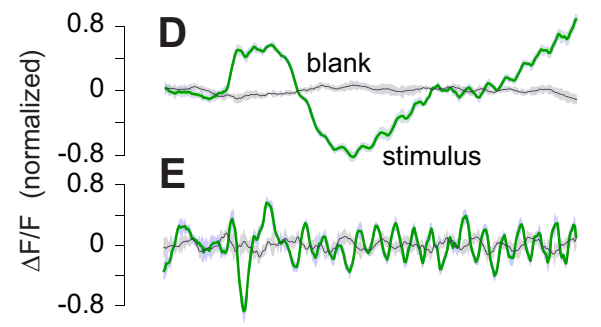

Raw

Filtered

$(1-10 \mathrm{~Hz})$

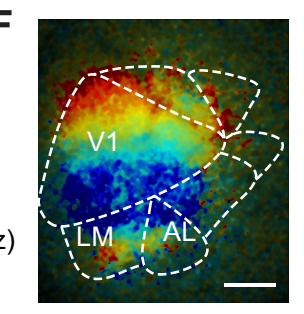

VSFP
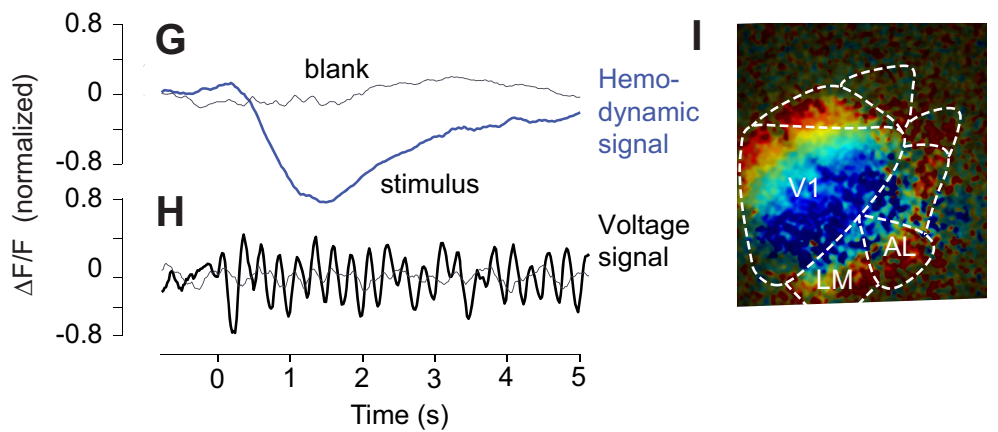

$\mathbf{J}$

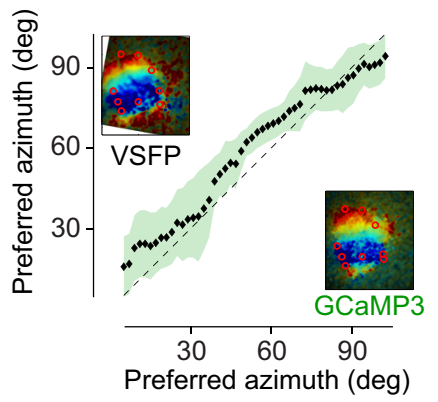

K

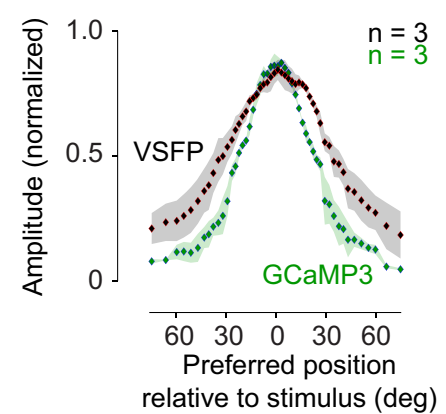

Figure 5. Comparison of VSFP with a calcium indicator, GCaMP3. Stimuli were vertical gratings reversing contrast at $2 \mathrm{~Hz}$ in narrow vertical windows ( $20^{\circ}$ wide), shown at four different horizontal positions. $A$, GCaMP3 showed high levels of expression in cortex. $\boldsymbol{B}$, The imaging window showed uniform fluorescence. $\boldsymbol{C}$, Confocal microscopy confirmed the localization of GCaMP3 in cortical neurons. $\boldsymbol{D}$, Raw GCaMP3 traces measured from a region in visual cortex. Units are normalized by peak response. Shaded area (barely visible) indicates $\pm 1 \mathrm{SE}$. $\boldsymbol{E}$, The same traces, bandpass filtered between 1 and $10 \mathrm{~Hz}$. F, Map of retinotopy obtained from second harmonic calcium signals. V1, LM, and AL are three cortical areas identified by varying the horizontal position (these data) and vertical position (data not shown) of the rectangular stimuli. Scale bar, $1 \mathrm{~mm}$. $\mathbf{G}, \boldsymbol{H}$, Hemodynamic signal (G) and voltage signal $(\boldsymbol{H})$ measured in mice expressing VSFP while using the same contrast-reversing stimuli. $\boldsymbol{I}$, Map of retinotopy obtained from the second harmonic amplitude of the voltage signal. $\boldsymbol{J}$, Consistency of maps of area V1 obtained with GCaMP3 $(\boldsymbol{F})$ and with VSFP (I). Maps were aligned on the basis of cortical vertical meridians and compared pixel by pixel. $\boldsymbol{K}$, Comparison of tuning width across maps measured with VSFP and GCaMP3.

responses. The hemodynamic signal, averaged over multiple stimulus trials to minimize the impact of heart beats, provided clear maps of retinotopy (Fig. $4 A, B$ ), similar to those seen when imaging vascular responses (Schuett et al., 2002; Kalatsky and Stryker, 2003; Pisauro et al., 2013). The voltage signal also gave clear maps of retinotopy (Fig. 4C,D). These were obtained by measuring the amplitude of the oscillating response at the second harmonic frequency (Benucci et al., 2007). Reassuringly, the maps provided by the two signals were highly consistent $\left(R^{2}=0.9\right.$; Fig. $\left.4 E\right)$. Similar results were obtained in three other mice.

The spatial spread of hemodynamic signals was not significantly larger than that of voltage signals. For each pixel, we fitted the response amplitudes for different stimulus positions (azimuth or elevation) with a Gaussian function. The position where the function peaks is the preferred position of that pixel (Fig. $4 B, D, E)$. The width $\sigma$ of the Gaussian function was a free but global parameter for the fit. The mean $( \pm \mathrm{SD})$ values of $\sigma$ appeared to be larger for the hemodynamic signal $\left(38.0 \pm 13.4^{\circ}\right)$ than for the voltage signal $\left(28.2 \pm 5.5^{\circ}\right)$, but the difference was not statistically significant ( $p=$ 0.35 , paired $t$ test; $n=4$ mice).

We conclude that the voltage signal measured by VSFP imaging in response to isolated visual stimuli is generally colocalized with the hemodynamic signal. More generally, VSFP imaging offers the possibility of measuring not only a neural signal with high temporal resolution, but also the associated hemodynamic activity, at the same time, and to compare the two across trials and stimulus conditions.

\section{Comparison with calcium signals}

Having demonstrated the use of a GEVI to measure population responses in the visual cortex of awake mice, we asked how it compares to an established genetically encoded indicator of calcium, GCaMP3 (Zariwala et al., 2012). We particularly sought to compare signal-to-noise ratios provided by the two indicators. Moreover, we expected to see some differences, as GEVIs measure subthreshold membrane potential, whereas calcium indicators measure spiking activity (Berger et al., 2007).

To image GCaMP3 activity, we used transgenic mice expressing this indicator in excitatory cortical neurons (Fig. 5A-C). We crossed transgenic mice expressing the calcium indicator GCaMP3 conditional on the presence of Cre (Zariwala et al., 2012) with mice in which Cre is present in cells expressing Emxl. These are excitatory neurons of the neocortex and hippocampus, and glial cells of the pallium (Gorski et al., 2002). As expected, we found high levels of expression of GCaMP3 in cortex (Fig. $5 A$ ), which resulted into a uniformly fluorescent imaging window (Fig. 5B). Confocal microscopy confirmed the localization of GCaMP3 in cortical neurons (Fig. 5C).

The GCaMP3 traces were heavily corrupted by vascular artifacts, which could be removed only by temporal filtering (Fig. 
$5 D-F)$. Indeed, GCaMP3 fluoresces only in one fluorescence band and was thus acquired with a single camera. As such, one cannot use a ratiometric or projection method to remove the vascular artifacts. The raw traces therefore showed slow variations over the time scale of seconds, which is typical of hemodynamic activity (Fig. 5D). Moreover, they showed fast oscillations at the frequency of the heart rate (visible in individual trials). The only way in which we could minimize these artifacts was to bandpass filter the traces in time (Fig. $5 E$ ), removing all signals below $0.5-1.0 \mathrm{~Hz}$ (to avoid the slow hemodynamic activity) and above $10-12 \mathrm{~Hz}$ (to avoid the heart rate artifact). This filtering does remove some of the neural activity — such as the initial, positive onset response-and it reflects a fundamental problem with wide-field imaging of calcium signals. However, it does not affect the second harmonic oscillation at $4 \mathrm{~Hz}$ caused by the contrastreversing gratings. We used the amplitude of this oscillation to obtain orderly maps of retinotopy from GCaMP3 signals (Fig. 5F).

To compare these results with those obtained with the GEVI, we imaged our mice expressing VSFP using the same contrastreversing stimuli. As expected, the projection method yielded clear separation of a hemodynamic signal (Fig. $5 G$ ) from a voltage signal (Fig. $5 H$ ), and the latter gave rise to high-quality maps of retinotopy (Fig. 5I). These maps appeared highly consistent with those measured in mice expressing GCaMP3. To get a sense of their similarity, we compared their progression along the horizontal axis in the visual field (azimuth). We aligned the maps obtained with the two methods, so that they would have the same representation of the vertical meridian (azimuth $=0^{\circ}$ ), and compared the preferred azimuths of each point in one map with the same point in the other map. The results indicate a very similar progression of preferred azimuth with cortical distance between the two maps (Fig. 5J). This similarity is reassuring, especially when considering that it is, by necessity, measured across different animals.

As expected, selectivity for stimulus position was sharper in GCaMP3 responses, which reflect spiking activity, than in VSFP responses, which reflect subthreshold activity (Fig. $5 \mathrm{~K}$ ). To compare the two, for each pixel we fitted a Gaussian position tuning curve to the amplitude of the responses to visual stimulation at four horizontal positions. The best SD $\sigma$ for the Gaussian curve model was chosen to be the one that minimized the least squared error of the fit for all pixels. Using this measure, we found that tuning curves were sharper when measured with GCaMP3 (mean $\pm \mathrm{SE}, \sigma=21.0 \pm 2.2 ; N=4)$ than with $\operatorname{VSFP}(\sigma=30.1 \pm$ $1.5 ; N=5$ ), a difference that was significant ( $t$ test, $p=0.019$ ). This result is consistent with well known differences in selectivity between subthreshold and spiking responses (Bringuier et al., 1999; Carandini and Ferster, 2000).

Finally, we compared the reliability for VSFP and GCaMP3 in terms of signal-to-noise ratios, and found them to be similar. As explained earlier, we measured the signal as the amplitude of the frequency response at twice the stimulus frequency (second harmonic) when a stimulus was present, and the noise as that amplitude when there was no stimulus (i.e., blank condition). When measured with VSFP, this signal-to-noise ratio averaged ( \pm SE) $11.8 \pm 2.8$ ( $n=3$ mice, average of $10-20$ trials $)$, compared with $12.0 \pm 1.8(n=4$ mice, average of 20 trials $)$ when measured with GCaMP3. The two were not significantly different $(p=0.95$, two-sample $t$ test).

\section{Space-time activation maps from voltage signals}

The maps that we have shown with VSFP are the result of extensive temporal averaging, involving multiple stimulus cycles in multiple trials. However, one of the most exciting prospects for

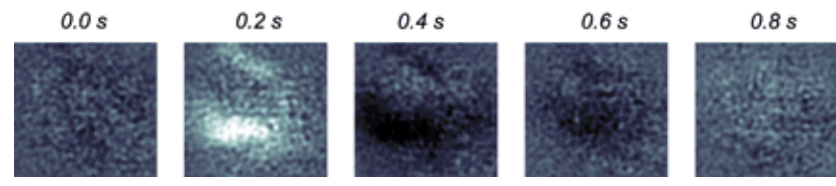

Movie 1. Average VSFP response to a flashed bar stimulus, with onset at $0 \mathrm{~s}$ and offset at $0.2 \mathrm{~s}$. These data are analyzed in Figure 6 . The upper graph shows the mean fluorescence within the red rectangle, in $\Delta F / F(\%)$, as a function of time, in seconds.

wide-field imaging lies in its ability to reveal the activity of large populations in single trials, or even in the absence of any stimulus (Arieli et al., 1996; Grinvald and Hildesheim, 2004; Mohajerani et al., 2010, 2013; Akemann et al., 2012; Scott et al., 2014). Indeed, the concept of "trial" is meaningful only for a repeatable sensory or motor protocol with a defined start time, not for endogenously generated activity associated with rest states or higher cognitive functions. Moreover, any attempt at decoding neural activity should be based on single trials, because the brain can rarely average across trials before making decisions. Therefore, it is essential for techniques for wide-field imaging to reveal activity in single trials.

To assess the ability of VSFP imaging to reveal activity in single trials, we considered the responses to brief visual stimuli, bar stimuli flashed for $200 \mathrm{~ms}$ (Fig. 2), and compared the individual responses with their average across 30 trials (Movie 1; Fig. 6). As we have seen, these brief stimuli produced a voltage signal with a characteristic biphasic profile (Fig. $6 A-D$ ), with a sharp increase (depolarization) followed by a decrease (hyperpolarization). The spatial profile of the depolarization (Fig. 6A, $0.2 \mathrm{~s}$ ) was similar to that of the subsequent hyperpolarization (Fig. $6 A, 0.4 \mathrm{~s}$ ). Indeed, the activity was well predicted by a simple separable model (Fig. $6 B-D$ ) where responses are simply the product of a spatial profile (Fig. 6C) and a temporal profile (Fig. 6D). In most experiments, the spatial profile included not only cortical area $\mathrm{V} 1$ but also area LM (Fig. 6C; Wang and Burkhalter, 2007), and in some experiments also higher visual areas. These results thus differ from those obtained in somatosensory cortex, where depolarization has been suggested to be followed by a wider hyperpolarization (Takashima et al., 2001; Devor et al., 2007).

The separable model is an effective but coarse summary of the activity over a relatively long time scale, and is not incompatible with subtler, more complex dynamics that have been observed at finer time scales in cats and primates (Sit et al., 2009; Sato et al., 2012). The advantage of the model is that it allowed us to define the responses in terms of a single spatial profile and a single temporal profile. This characteristic proved particularly useful when analyzing single trials, as we see next. VSFP signals were sufficiently strong to reveal responses in individual trials, and revealed that activity could differ substantially across trials. In some trials, activity resembled the average across trials both in terms of spatial profile and in terms of temporal profile, whereas in other trials it tended to differ in both respects (Fig. 6E). An examination of a representative set of trials further illustrates this phenomenon (Fig. $6 F, G$ ). In some trials, such as the 11 th, 7 th, and 12th, both spatial and temporal profiles resembled those measured, on average (Fig. 6C,D). In other trials, such as the 10th and the 2nd, responses differed both in spatial profile and in temporal profile. Given that VSFP gives reliable responses to direct neuronal stimulation in vitro (Akemann et al., 2010, 2012), these differences across trials are likely to reflect genuine variations in neural activation across space and time. These may be due to differences in ongoing activity at the onset of each stimulus 
A
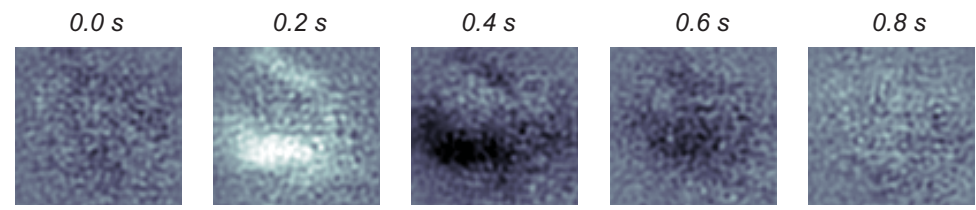

B
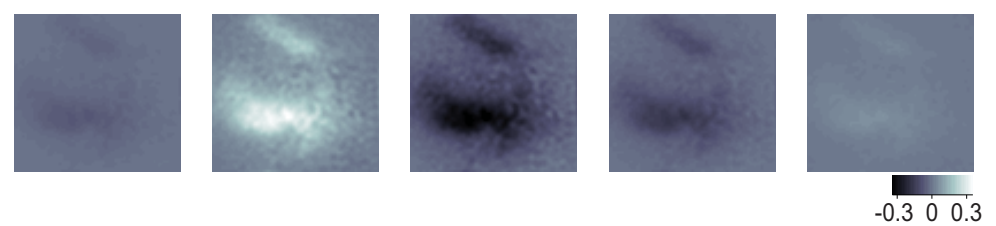

C
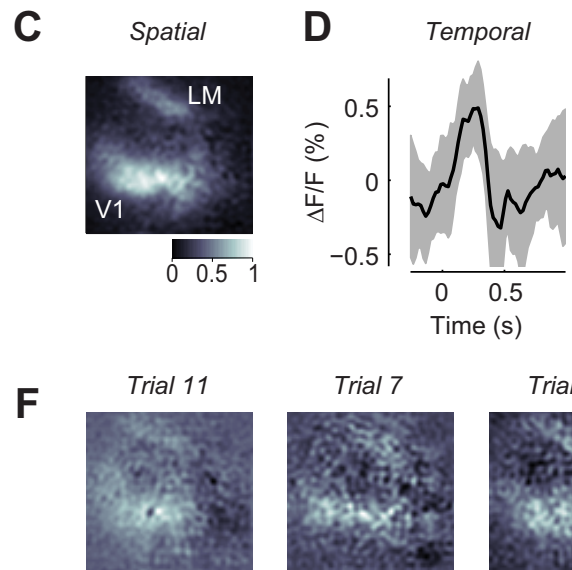

G
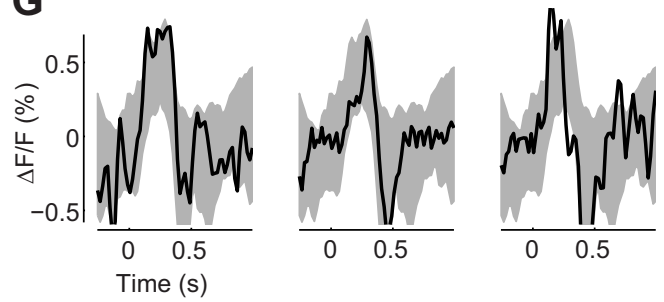

1.0 E

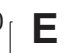
$\Delta \mathrm{F} / \mathrm{F}(\%)$

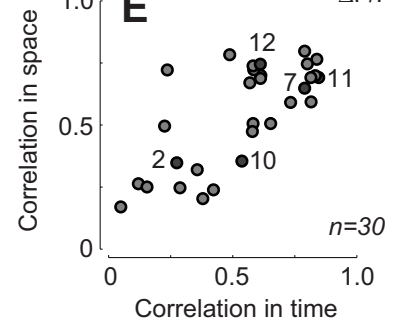

Trial 7

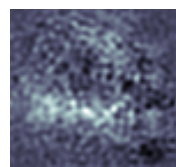

Trial 12

Trial 10
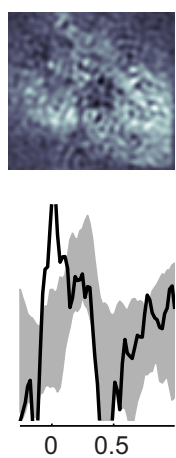

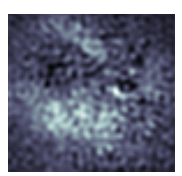

Trial 2

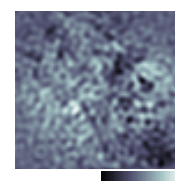

$0 \quad 1$

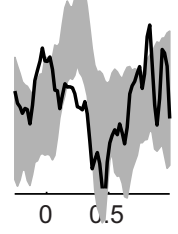

Figure 6. Spatial extent of VSFP responses to a flashed stimulus, and quality of single-trial responses. $A$, Spatial profile of activity at five intervals relative to a flashed bar with onset at $0 \mathrm{~s}$ and offset at $0.2 \mathrm{~s}$, averaged over 30 stimulus repetitions. The "salt-and-pepper" appearance of these images is due to photon noise, which is only partially reduced by smoothing. $\boldsymbol{B}$, Separable approximation of the responses, evaluated at the same five intervals. This approximation is the product of a single spatial profile and a single temporal profile. The scale bar applies to all panels in $\boldsymbol{A}$ and $\boldsymbol{B}$. $\boldsymbol{C}$, The spatial profile, which includes activations in two visual areas, $\mathrm{V} 1$ and $\mathrm{LM}$. Scale is normalized so that the maximum is 1 . $\boldsymbol{D}$, The temporal profile. Gray region indicates \pm 1 SD over 30 stimulus repetitions. The scale is such that when multiplied with the image in $\boldsymbol{C}$, it yields responses in $\boldsymbol{B}$. $\boldsymbol{E}$, Repeatability of spatial profile and temporal profile in individual trials. In some trials, the temporal profile and spatial profile correlated with the average (shown in $\boldsymbol{C}$ and $\boldsymbol{D}$ ) more than in others. Five trials are marked as examples as they divide the sample into quintiles. $\boldsymbol{F}, \boldsymbol{G}$, Spatial and temporal profiles of activity in those five trials, arranged by order of decreasing similarity with the average responses (left to right).

(Arieli et al., 1996; Petersen et al., 2003; Curto et al., 2009). Further research will be required to elucidate this and other questions, and our results indicate that this research can be fruitfully pursued using wide-field imaging of voltage-sensitive fluorescent proteins.

\section{Discussion}

We have shown that VSFP-Butterfly 1.2, a GEVI, can be fruitfully used to reveal the membrane potential of neuronal populations across large portions of the mouse visual cortex during wakefulness. VSFP-Butterfly 1.2 offers numerous advantages, including approximate linearity of responses in the range of typical subthreshold membrane potentials, and good temporal resolution (Akemann et al., 2010, 2012). Here we showed that it can be used effectively during wakefulness, a condition that is more challenging than under anesthesia, because membrane potential responses are typically faster (Haider et al., 2013) and confounding hemodynamic activity is stronger (Pisauro et al., 2013).

We delivered the indicator through in utero electroporation, thus obtaining strong and selective expression in layer $2 / 3$ pyramidal cells. This method of delivery allows highly selective targeting of pyramidal cells in a layer of choice. This selectivity overcomes the promiscuity of the CAG promoter, which drives gene expression in a wide range of cell types, including astrocytes. Indeed, the cells that line the lateral ventricle at embryonic day 15.5 migrate during development to become primarily layer $2 / 3$ pyramidal cells. Astrocytes, instead, migrate into cortex from a different site, which is not targeted by the electroporation.

To fully exploit the signals obtained from the indicator, we developed a straightforward projection method that separates its outputs into a signal dominated by voltage and a signal dominated by hemodynamics. This method exploits the dual emission of the FRET-based voltage indicator, which results in two fluorescent traces. Voltage signals are traditionally estimated by taking the ratio of the two traces (Akemann et al., 2012; Knöpfel, 2012). Our alternative method is based on linear projection: it simply takes the signals from both of the fluorophores of the indicator and computes weighted sums of the two measurements. This method estimates not only voltage signals (similar to those obtained by taking the ratios), but also hemodynamic signals.

The resulting voltage and hemodynamic signals were well separated in both the temporal domain and the frequency domain. The hemodynamic signal contains strong oscillations due to the heartbeat, which were almost entirely absent in the voltage signal. Superimposed on this fast oscillation is a slower neurovascular response similar to BOLD responses measured in awake mice with functional MRI (Desai et al., 2011) and with optical imaging (Pisauro et al., 2013). The voltage signals, in turn, were markedly larger than single-trial measurement noise and had fast dynamics, similar to those observed with intracellular recordings in awake mice (Haider et al., 2013) and broadly comparable to measurements made with classic voltage-sensitive dyes (Polack and Contreras, 2012; Mohajerani et al., 2013).

One possible confound when measuring fluorescence in the brain is the presence of activity-dependent fluorescence from mitochondrial flavoproteins. This autofluorescence is robust and can be used to obtain maps of activation (Husson et al., 2007; Andermann et al., 2011; Michael et al., 2014). It is a potential source of undesired signal components when using fluorescent 
indicators with emission in the 500-600 $\mathrm{nm}$ range (Coutinho et al., 2004; Díez-García et al., 2007). However, there are multiple reasons to conclude that autofluorescence can be neglected when interpreting the voltage and hemodynamic signals that we have obtained in this study. First, the fluorescence of the brain outside the regions expressing the indicator is negligible: those regions are extremely dark (Fig. 1A). Second, the autofluorescence signal would have the same polarity in both channels (mostly, in the green channel), whereas the voltage signal is opposite in the two channels. Third, the time course of both voltage and hemodynamic signals is faster than would be expected from autofluorescence. For instance, the hemodynamic signal faithfully follows the heartbeats, which would not be visible in autofluorescence imaging. These observations indicate that autofluorescence does not make a relevant contribution to the signals that we analyzed.

Another limitation of our analysis is that it treats the vascular effects as a time-varying neutral density filter. This is a simplification, justified empirically by our finding reasonably good separation between the two signals produced by the projection method. It is, nonetheless, a simplification, because the ratio of oxyhemoglobin $(\mathrm{HbO})$ to deoxyhemoglobin $(\mathrm{Hb})$ is thought to change during the course of a neural response and afterward, and also during ongoing activity. While the donor fluorescence wavelength $(542 \mathrm{~nm})$ is slightly less absorbed by $\mathrm{Hb}$ and $\mathrm{HbO}$ (by a factor of 0.9 ), the acceptor fluorescence wavelength $(594 \mathrm{~nm})$ is 2.9 times more strongly absorbed by $\mathrm{Hb}$ than by $\mathrm{HbO}$ (Prahl, 1999). Thus, as the proportion of $\mathrm{Hb}$ and $\mathrm{HbO}$ changes in cortex, the fractional absorption by hemodynamics would change at these two wavelengths, differentially affecting donor and acceptor signals. A more precise measurement would be obtained with a third camera, which could image at a wavelength where $\mathrm{Hb}$ and $\mathrm{HbO}$ are equally absorbed (isosbestic wavelength; Kramer and Pearlstein, 1979). Alternatively, one could combine fluorescence imaging and reflectance imaging (e.g., in alternate camera frames; Bouchard et al., 2009).

We found that the voltage signal and the hemodynamic signal provided congruent maps of retinotopy, with an approximately similar spatial footprint. This similarity had been suggested using VSD imaging (Takashima et al., 2001), and it has implications for the spatial footprint of BOLD signals. There is debate as to whether these signals reveal mostly spiking activity (Heeger et al., 2000) or subthreshold activity (Logothetis and Wandell, 2004). The activations measured by imaging voltage signals are certainly wider than those corresponding to spiking activity (Grinvald and Hildesheim, 2004). Their resemblance to those obtained from hemodynamic signals suggests that the latter indeed reflect subthreshold neural activation more than spiking activity. Indeed, we found the spatial footprint of spiking activity to be narrower than that of the voltage signal (Fig. 5).

Our analysis demonstrates that voltage imaging using GEVIs is sufficiently developed to illuminate the organization and dynamics of neural circuits at mesoscopic spatial scales and at a wide range of temporal scales, from tens of milliseconds to weeks. We have not probed the temporal resolution of the GEVI at shorter time scales, because of the limitations inherent in the visual system, which do not follow fast changes. An ideal test bench for probing temporal resolution is the somatosensory cortex, which allows finer temporal control of neuronal activity (Ferezou et al., 2006, 2007; Berger et al., 2007). Therefore, VSFP-Butterfly 1.2 has been shown to reveal activity with good temporal resolution (Akemann et al., 2012).

GEVIs present multiple advantages over traditional VSDs (Knöpfel, 2012). GEVIs allow genetic targeting to specific cell populations, providing a handle into cellular diversity, and they allow reliable recordings from those populations over prolonged periods and multiple sessions. Moreover, using a GEVI we could perform the imaging in awake animals, following a simple surgical procedure that did not involve a craniotomy. Along with the potential pharmacological side effects of VSDs (Mennerick et al., 2010; Grandy et al., 2012), this highlights the clear advantages of the GEVI approach.

The voltage signals were much larger than the measurement noise, allowing for single-trial imaging and for repeated measurements across multiple weeks, and were as reliable as estimates obtained with a GECI. Because GECIs mostly reveal the activity due to spiking, performing the same experiments with GEVIs and GECIs allows one to distinguish the activations that correspond to spiking alone from those that correspond also to subthreshold inputs, including hyperpolarizing inputs (Minderer et al., 2012). GECIs currently hold the advantage of allowing two-photon imaging, but this advantage may be partially eroding, as two-photon imaging is beginning to be used also with GEVIs (Akemann et al., 2013).

Together, these results demonstrate that the prospects of mesoscopic imaging of GEVIs are bright. GEVIs provide a powerful experimental approach to study the dynamics of neural circuits at mesoscopic spatial scales and millisecond temporal scales. Their advantages will only become stronger once transgenic mice that carry the VSFP Butterfly 1.2 gene in a Cre-dependent configuration become available. Preliminary results indicate that the voltage signals from these transgenic mice are as good as those in the mice presented in this study (Shimaoka et al., 2014). By enabling a delivery that is noninvasive, highly reproducible, genetically targeted, and spatially widespread, these transgenic mice promise to unleash the full potential of genetically encoded voltage indicators.

\section{References}

Akemann W, Mutoh H, Perron A, Rossier J, Knöpfel T (2010) Imaging brain electric signals with genetically targeted voltage-sensitive fluorescent proteins. Nat Methods 7:643-649. CrossRef Medline

Akemann W, Mutoh H, Perron A, Park YK, Iwamoto Y, Knöpfel T (2012) Imaging neural circuit dynamics with a voltage-sensitive fluorescent protein. J Neurophysiol 108:2323-2337. CrossRef Medline

Akemann W, Sasaki M, Mutoh H, Imamura T, Honkura N, Knöpfel T (2013) Two-photon voltage imaging using a genetically encoded voltage indicator. Sci Rep 3:2231. CrossRef Medline

Andermann ML, Kerlin AM, Roumis DK, Glickfeld LL, Reid RC (2011) Functional specialization of mouse higher visual cortical areas. Neuron 72:1025-1039. CrossRef Medline

Arieli A, Sterkin A, Grinvald A, Aertsen A (1996) Dynamics of ongoing activity: explanation of the large variability in evoked cortical responses. Science 273:1868-1871. CrossRef Medline

Ayaz A, Saleem AB, Schölvinck ML, Carandini M (2013) Locomotion controls spatial integration in mouse visual cortex. Curr Biol 23:890-894. CrossRef Medline

Benucci A, Frazor RA, Carandini M (2007) Standing waves and traveling waves distinguish two circuits in visual cortex. Neuron 55:103-117. CrossRef Medline

Berger T, Borgdorff A, Crochet S, Neubauer FB, Lefort S, Fauvet B, Ferezou I, Carleton A, Lüscher HR, Petersen CC (2007) Combined voltage and calcium epifluorescence imaging in vitro and in vivo reveals subthreshold and suprathreshold dynamics of mouse barrel cortex. J Neurophysiol 97:3751-3762. CrossRef Medline

Bouchard MB, Chen BR, Burgess SA, HIllmann EM (2009) Ultra-fast multispectral optical imaging of cortical oxygenation, blood flow, and intracellular calcium dynamics. Opt Express 17:15670-15678. CrossRef Medline

Bringuier V, Chavane F, Glaeser L, Frégnac Y (1999) Horizontal propagation of visual activity in the synaptic integration field of area 17 neurons. Science 283:695-699. CrossRef Medline

Carandini M, Ferster D (2000) Membrane potential and firing rate in cat primary visual cortex. J Neurosci 20:470-484. Medline

Coutinho V, Mutoh H, Knöpfel T (2004) Functional topology of the mossy fibre-granule cell-Purkinje cell system revealed by imaging of intrinsic 
fluorescence in mouse cerebellum. Eur J Neurosci 20:740-748. CrossRef Medline

Curto C, Sakata S, Marguet S, Itskov V, Harris KD (2009) A simple model of cortical dynamics explains variability and state dependence of sensory responses in urethane-anesthetized auditory cortex. J Neurosci 29: 10600-10612. CrossRef Medline

Desai M, Kahn I, Knoblich U, Bernstein J, Atallah H, Yang A, Kopell N, Buckner RL, Graybiel AM, Moore CI, Boyden ES (2011) Mapping brain networks in awake mice using combined optical neural control and fMRI. J Neurophysiol 105:1393-1405. CrossRef Medline

Devor A, Tian P, Nishimura N, Teng IC, Hillman EM, Narayanan SN, Ulbert I, Boas DA, Kleinfeld D, Dale AM (2007) Suppressed neuronal activity and concurrent arteriolar vasoconstriction may explain negative blood oxygenation level-dependent signal. J Neurosci 27:4452-4459. CrossRef Medline

Díez-García J, Akemann W, Knöpfel T (2007) In vivo calcium imaging from genetically specified target cells in mouse cerebellum. Neuroimage 34: 859-869. CrossRef Medline

Dombeck DA, Khabbaz AN, Collman F, Adelman TL, Tank DW (2007) Imaging large-scale neural activity with cellular resolution in awake, mobile mice. Neuron 56:43-57. CrossRef Medline

Ferezou I, Bolea S, Petersen CC (2006) Visualizing the cortical representation of whisker touch: voltage-sensitive dye imaging in freely moving mice. Neuron 50:617-629. CrossRef Medline

Ferezou I, Haiss F, Gentet LJ, Aronoff R, Weber B, Petersen CC (2007) Spatiotemporal dynamics of cortical sensorimotor integration in behaving mice. Neuron 56:907-923. CrossRef Medline

Fukuchi-Shimogori T, Grove EA (2001) Neocortex patterning by the secreted signaling molecule FGF8. Science 294:1071-1074. CrossRef Medline

Gorski JA, Talley T, Qiu M, Puelles L, Rubenstein JL, Jones KR (2002) Cortical excitatory neurons and glia, but not GABAergic neurons, are produced in the Emx1-expressing lineage. J Neurosci 22:6309-6314. Medline

Grandy TH, Greenfield SA, Devonshire IM (2012) An evaluation of in vivo voltage-sensitive dyes: pharmacological side effects and signal-to-noise ratios after effective removal of brain-pulsation artifacts. J Neurophysiol 108:2931-2945. CrossRef Medline

Grinvald A, Hildesheim R (2004) VSDI: a new era in functional imaging of cortical dynamics. Nat Rev Neurosci 5:874-885. CrossRef Medline

Haider B, Häusser M, Carandini M (2013) Inhibition dominates sensory responses in the awake cortex. Nature 493:97-100. CrossRef Medline

Hazan L, Zugaro M, Buzsáki G (2006) Klusters, NeuroScope, NDManager: a free software suite for neurophysiological data processing and visualization. J Neurosci Methods 155:207-216. CrossRef Medline

Heeger DJ, Huk AC, Geisler WS, Albrecht DG (2000) Spikes versus BOLD: what does neuroimaging tell us about neuronal activity? Nat Neurosci 3:631-633. CrossRef Medline

Hillman EM (2007) Optical brain imaging in vivo: techniques and applications from animal to man. J Biomed Opt 12:051402. CrossRef Medline

Husson TR, Mallik AK, Zhang JX, Issa NP (2007) Functional imaging of primary visual cortex using flavoprotein autofluorescence. J Neurosci 27:8665-8675. CrossRef Medline

Jares-Erijman EA, Jovin TM (2003) FRET imaging. Nat Biotechnol 21: 1387-1395. CrossRef Medline

Kalatsky VA, Stryker MP (2003) New paradigm for optical imaging: temporally encoded maps of intrinsic signal. Neuron 38:529-545. CrossRef Medline

Knöpfel T (2012) Genetically encoded optical indicators for the analysis of neuronal circuits. Nat Rev Neurosci 13:687-700. CrossRef Medline

Kramer RS, Pearlstein RD (1979) Cerebral cortical microfluorometry at isosbestic wavelengths for correction of vascular artifact. Science 205: 693-696. CrossRef Medline

Logothetis NK, Wandell BA (2004) Interpreting the BOLD signal. Annu Rev Physiol 66:735-769. CrossRef Medline

Marshel JH, Garrett ME, Nauhaus I, Callaway EM (2011) Functional specialization of seven mouse visual cortical areas. Neuron 72:1040-1054. CrossRef Medline

Mennerick S, Chisari M, Shu HJ, Taylor A, Vasek M, Eisenman LN, Zorumski CF (2010) Diverse voltage-sensitive dyes modulate GABAA receptor function. J Neurosci 30:2871-2879. CrossRef Medline

Michael N, Bischof HJ, Löwel S (2014) Flavoprotein autofluorescence im- aging of visual system activity in zebra finches and mice. PLoS One 9:e85225. CrossRef Medline

Minderer M, Liu W, Sumanovski LT, Kügler S, Helmchen F, Margolis DJ (2012) Chronic imaging of cortical sensory map dynamics using a genetically encoded calcium indicator. J Physiol 590:99-107. CrossRef Medline

Mizuno H, Hirano T, Tagawa Y (2007) Evidence for activity-dependent cortical wiring: formation of interhemispheric connections in neonatal mouse visual cortex requires projection neuron activity. J Neurosci 27: 6760-6770. CrossRef Medline

Mohajerani MH, McVea DA, Fingas M, Murphy TH (2010) Mirrored bilateral slow-wave cortical activity within local circuits revealed by fast bihemispheric voltage-sensitive dye imaging in anesthetized and awake mice. J Neurosci 30:3745-3751. CrossRef Medline

Mohajerani MH, Chan AW, Mohsenvand M, LeDue J, Liu R, McVea DA, Boyd JD, Wang YT, Reimers M, Murphy TH (2013) Spontaneous cortical activity alternates between motifs defined by regional axonal projections. Nat Neurosci 16:1426-1435. CrossRef Medline

Movshon JA, Thompson ID, Tolhurst DJ (1978) Nonlinear spatial summation in the receptive fields of complex cells in the cat striate cortex. J Physiol (London) 283:78-100.

Petersen CC, Hahn TT, Mehta M, Grinvald A, Sakmann B (2003) Interaction of sensory responses with spontaneous depolarization in layer $2 / 3$ barrel cortex. Proc Natl Acad Sci U S A 100:13638-13643. CrossRef Medline

Pisauro MA, Dhruv NT, Carandini M, Benucci A (2013) Fast hemodynamic responses in the visual cortex of the awake mouse. J Neurosci 33:1834318351. CrossRef Medline

Polack PO, Contreras D (2012) Long-range parallel processing and local recurrent activity in the visual cortex of the mouse. J Neurosci 32:1112011131. CrossRef Medline

Prahl S (1999) Optical absorption of hemoglobin. Portland, OR: Oregon Medical Laser Center.

Romoser VA, Hinkle PM, Persechini A (1997) Detection in living cells of $\mathrm{Ca} 2+$-dependent changes in the fluorescence emission of an indicator composed of two green fluorescent protein variants linked by a calmodulin-binding sequence. A new class of fluorescent indicators. J Biol Chem 272:13270-13274. CrossRef Medline

Saito T, Nakatsuji N (2001) Efficient gene transfer into the embryonic mouse brain using in vivo electroporation. Dev Biol 240:237-246. CrossRef Medline

Sato TK, Nauhaus I, Carandini M (2012) Traveling waves in visual cortex. Neuron 75:218-229. CrossRef Medline

Sato TK, Häusser M, Carandini M (2014) Distal connectivity causes summation and division across mouse visual cortex. Nat Neurosci 17:30-32. CrossRef Medline

Schuett S, Bonhoeffer T, Hübener M (2002) Mapping retinotopic structure in mouse visual cortex with optical imaging. J Neurosci 22:6549-6559. Medline

Scott G, Fagerholm ED, Mutoh H, Leech R, Sharp DJ, Shew WL, Knöpfel (2014) Voltage imaging of waking mouse cortex reveals emergence of critical neuronal dynamics. J Neurosci 34:16611-16620. CrossRef

Shimaoka D, Benucci A, Knöpfel T, Carandini M, Zeng H (2014) Imaging cortical activity in a transgenic mouse expressing a voltage indicator. Paper presented at the 9th FENS Forum of Neuroscience, Milan, Italy, July.

Sit YF, Chen Y, Geisler WS, Miikkulainen R, Seidemann E (2009) Complex dynamics of $\mathrm{V} 1$ population responses explained by a simple gain-control model. Neuron 64:943-956. CrossRef Medline

Takashima I, Kajiwara R, Iijima T (2001) Voltage-sensitive dye versus intrinsic signal optical imaging: comparison of optically determined functional maps from rat barrel cortex. Neuroreport 12:2889-2894. CrossRef Medline

Van den Bergh G, Zhang B, Arckens L, Chino YM (2010) Receptive-field properties of V1 and V2 neurons in mice and macaque monkeys. J Comp Neurol 518:2051-2070. CrossRef Medline

Wang Q, Burkhalter A (2007) Area map of mouse visual cortex. J Comp Neurol 502:339-357. CrossRef Medline

Zariwala HA, Borghuis BG, Hoogland TM, Madisen L, Tian L, De Zeeuw CI, Zeng H, Looger LL, Svoboda K, Chen TW (2012) A Cre-dependent GCaMP3 reporter mouse for neuronal imaging in vivo. J Neurosci 32: 3131-3141. CrossRef Medline 